\title{
ON THE THEORY OF MOVING TYPE IV RADIO BURSTS
}

\author{
A. MANGENEY \\ Observatoire de Meudon, France
}

\begin{abstract}
Astron. Astrophys.). In this paper it is shown that the Alfvén wave turbulence created behind a shock wave propagating along the ambiant magnetic field in a collision-less plasma, accelerates electrons of the high energy tail of the distribution up to mildly relativistic energies. The efficiency of the process depends upon the ratio of the acceleration time by the turbulence to the scattering time of the same turbulence. At high energies the accelerated particles have a power law spectrum, the steepness of which depends upon the turbulent energy.

It is suggested that moving type IV radio bursts are due to the synchrotron emission of the accelerated electrons produced when a flare induced shock wave propagates along open field lines in the solar corona.
\end{abstract}

\section{DISCUSSION}

Smith: You say that your model satisfactorily explains the association of type IV to type II bursts. What do you think determines whether a shock produces a type II or type IV burst?

Mangeney: The configuration of shock and piston. 